\title{
Connecting Majorana phases to the geometric parameters of the Majorana unitarity triangle in a neutrino mass matrix model
}

\author{
Surender Verma* and Shankita Bhardwaj ${ }^{\dagger}$ \\ Department of Physics and Astronomical Science, Central University of Himachal Pradesh, \\ Dharamshala 176215, India
}

(Received 9 January 2018; published 17 May 2018)

\begin{abstract}
We have investigated a possible connection between the Majorana phases and geometric parameters of Majorana unitarity triangle (MT) in two-texture zero neutrino mass matrix. Such analytical relations can, also, be obtained for other theoretical models viz. hybrid textures, neutrino mass matrix with vanishing minors and have profound implications for geometric description of $C P$ violation. As an example, we have considered the two-texture zero neutrino mass model to obtain a relation between Majorana phases and MT parameters that may be probed in various lepton number violating processes. In particular, we find that Majorana phases depend on only one of the three interior angles of the MT in each class of two-texture zero neutrino mass matrix. We have also constructed the MT for class $A, B$, and $C$ neutrino mass matrices. Nonvanishing areas and nontrivial orientations of these Majorana unitarity triangles indicate nonzero $C P$ violation as a generic feature of this class of mass models.
\end{abstract}

DOI: 10.1103/PhysRevD.97.095022

\section{INTRODUCTION}

Recent developments in experimental neutrino physics have catalyzed assiduous efforts to understand the origin of neutrino mass. The neutrino oscillation experiments have, now, revealed that the dominant structure of the neutrino mixing matrix [1-4] and oscillation parameters such as $\theta_{12}$, $\theta_{23}, \theta_{13}, \Delta m_{21}^{2},\left|\Delta m_{31}^{2}\right|$ are known to an unprecedented accuracy. Besides these astonishing discoveries, there remain arduous questions viz., are neutrinos Majorana or Dirac particle? What is the lightest neutrino mass? Is neutrino mass hierarchy normal or inverted? What is the octant of the atmospheric mixing angle? And what is the leptonic $C P$ violating phase $\delta$ ? This is just to name a few. The primary goal of the future neutrino experimental endeavours will be to address these questions by employing a miscellany of experimental configurations and techniques.

The observation of the nonzero value of reactor mixing angle $\theta_{13}$ [5], by oscillation experiments, provides a unique opportunity for the possible measurement of $C P$ violation

\footnotetext{
*sverma@cuhimachal.ac.in

s_7verma@yahoo.co.in

shankita.bhardwaj982@gmail.com

Published by the American Physical Society under the terms of the Creative Commons Attribution 4.0 International license. Further distribution of this work must maintain attribution to the author(s) and the published article's title, journal citation, and DOI. Funded by SCOAP .
}

in the leptonic sector. The flavor oscillations imply the existence of mixing in the weak charged current interaction

$$
\begin{aligned}
\mathcal{L}_{C C} & =-\frac{g}{\sqrt{2}} \sum_{l=e, \mu, \tau} \bar{l}_{L}(x) \gamma_{\alpha} \nu_{l L}(x) W^{\alpha \dagger}(x)+\text { H.c. }, \\
\nu_{l L}(x) & =\sum_{j=1}^{n} U_{l j} \nu_{j L}(x),
\end{aligned}
$$

where, $\nu_{l L}(x)$ are flavor fields, $\nu_{j L}(x)$ are $L H$ component of mass fields $\nu_{j}$ with mass $m_{j}$, and $U$ is unitary mixing matrix. The mixing matrix $U$ can be parametrized in terms of $\frac{1}{2} n(n-1)$ angles and $\frac{1}{2}(n-1)(n-2)$ phases. For Majorana neutrinos, there exist $(n-1)$ additional $C P$ violating phases called "Majorana phases" [6]. In the standard framework of three-neutrino the mixing matrix contains two $C P$ violating phases $\rho$ and $\sigma$, in addition to one Dirac-type $C P$ violating phase $\delta$. While the Dirac-type $C P$ violating phase will, possibly, be measured in the neutrino oscillation experiments $\mathrm{T} 2 \mathrm{~K}, \mathrm{NO} \nu \mathrm{A}$, and DUNE, the information about Majorana-type $C P$ violating phases can be extracted from the lepton number violating (LNV) processes.

$C P$ violation in the leptonic sector can either be studied through the construction of the leptonic unitarity triangle or by direct measurement of the $C P$ violating phase $\delta$ in the neutrino oscillation experiments. The first approach has the advantage of being the rephasing invariant description of the $C P$ violation. 
In the flavor basis, the unitary mixing matrix $V \equiv U P$, where $U$ is Pontecorvo-Maki-Nakagawa-Sakata (PMNS) matrix, is given by

$$
U=\left(\begin{array}{ccc}
c_{12} c_{13} & s_{12} c_{13} & s_{13} e^{-i \delta} \\
-s_{12} c_{23}-c_{12} s_{23} s_{13} e^{i \delta} & c_{12} c_{23}-s_{12} s_{23} s_{13} e^{i \delta} & s_{23} c_{13} \\
s_{12} s_{23}-c_{12} c_{23} s_{13} e^{i \delta} & -c_{12} s_{23}-s_{12} c_{23} s_{13} e^{i \delta} & c_{23} c_{13}
\end{array}\right)
$$

$s_{i j}=\sin \theta_{i j}, \quad c_{i j}=\cos \theta_{i j}, \quad$ and $P=\operatorname{diag}\left(1, e^{i \rho}, e^{i(\sigma+\delta)}\right)$. The unitarity of $V$ imposes six orthogonality conditions on the elements of $V$ viz.,

$$
\begin{aligned}
\Delta_{e \mu} & \equiv V_{e 1} V_{\mu 1}^{*}+V_{e 2} V_{\mu 2}^{*}+V_{e 3} V_{\mu 3}^{*}=0 \\
\Delta_{\mu \tau} & \equiv V_{\mu 1} V_{\tau 1}^{*}+V_{\mu 2} V_{\tau 2}^{*}+V_{\mu 3} V_{\tau 3}^{*}=0 \\
\Delta_{\tau e} & \equiv V_{\tau 1} V_{e 1}^{*}+V_{\tau 2} V_{e 2}^{*}+V_{\tau 3} V_{e 3}^{*}=0
\end{aligned}
$$

obtained from multiplying two rows of $V$ and

$$
\begin{aligned}
& \Delta_{12} \equiv V_{e 1} V_{e 2}^{*}+V_{\mu 1} V_{\mu 2}^{*}+V_{\tau 1} V_{\tau 2}^{*}=0 \\
& \Delta_{23} \equiv V_{e 2} V_{e 3}^{*}+V_{\mu 2} V_{\mu 3}^{*}+V_{\tau 2} V_{\tau 3}^{*}=0, \\
& \Delta_{31} \equiv V_{e 3} V_{e 1}^{*}+V_{\mu 3} V_{\mu 1}^{*}+V_{\tau 3} V_{\tau 1}^{*}=0
\end{aligned}
$$

obtained from multiplying two columns of $V$. In the complex plane, Eq. (3) describes the "Dirac triangles (DT)" whereas Eq. (4) describes "Majorana triangles (MT)" [7]. Under the rephasing transformations of the leptonic fields, the mixing matrix $V$ transforms as $V_{l i} \rightarrow e^{i \phi_{i}} V_{l i}$. Thus, the Dirac unitarity conditions, Eq. (3), rotate in the complex plane and their orientations have no physical significance. In general, the vanishing area of DT does not, necessarily, imply vanishing $C P$ violation because of the nonzero contribution coming from the Majorana sector. The orientations of Majorana unitarity triangles are physical because, as can be seen from Eq. (4), they correspond to the sum of bilinear rephasing invariant terms. Thus, Majorana triangles provide a complete description of the $C P$ violation.

Although the dominant structure of the PMNS matrix has been revealed, $C P$ violating phases are still unknown. There are various theoretical constructions to restrict the number of free parameters in the leptonic flavor sector to make a particular model of neutrino masses and mixings more predictive. These approaches include texture zeros [8-11], hybrid texture [12,13], and vanishing minor [14,15], which are consistent with current data on neutrino masses and mixings. These Ansatze have profound phenomenological implications for our quest to understand the origin of neutrino masses and mixings. In the present work, we have considered a two-texture zero model of neutrino mass matrix and derive possible connection between the Majorana phases and geometric parameters of the Majorana unitarity triangle.
In Sec. II, we have reviewed the two-texture zero mass model and obtained mass ratios to study the complete phenomenology of the model. We derive a relation between Majorana phases and geometric parameters of Majorana unitarity triangles, and construct these triangles for all viable two-texture zero neutrino mass matrices in Sec. III. In Secs. IV and V, we have investigated the status of $0 \nu \beta \beta$ decay and observable sensitive to geometric parameters of MT, respectively, in two-texture zero neutrino mass models. In Sec. VI we have discussed the generic features of the MTs and, finally, we conclude in Sec. VII.

\section{TWO-TEXTURE ZEROS NEUTRINO MASS MATRICES}

In the flavor basis, where the charged-lepton mass matrix $M_{\nu}$ is diagonal, the Majorana neutrino mass matrix $M$ is given by

$$
M=V M_{\nu} V^{T},
$$

where $M_{\nu}=\operatorname{Diag}\left\{m_{1}, m_{2}, m_{3}\right\}$. There are a total of 15 possible patterns of two-texture zero Majorana neutrino mass matrices. Seven out of these patterns, are found to be consistent with the neutrino mixing parameters. These are $A_{1}, A_{2}, B_{1}, B_{2}, B_{3}, B_{4}$, and $C$ (Table I). Since $M$ is symmetric it has total six independent complex elements. Any two vanishing elements of $M$, i.e., $M_{s t}=0, M_{x y}=0$, where $s, t, x$, and $y$ can take values $e, \mu, \tau$, result in two complex constraining equations, viz., $\sum_{i=1}^{3} V_{s i} V_{t i} m_{i}=0$, $\sum_{i=1}^{3} V_{x i} V_{y i} m_{i}=0$. These two relations involve nine free parameters, i.e., $m_{1}, m_{2}, m_{3}, \theta_{12}, \theta_{13}, \theta_{23}$ and three $C P$ violating phases $\rho, \sigma$, and $\delta$, where $\rho, \sigma$ are two Majoranatype $C P$ violating phases and $\delta$ is a Dirac-type $C P$ violating phase. We solve these constraining equations to obtain mass ratios $\left(\frac{m_{1}}{m_{2}}, \frac{m_{1}}{m_{3}}\right)$ and Majorana phases $(\rho, \sigma)$ as

$$
\begin{aligned}
& \frac{m_{1}}{m_{2}}=\left|\frac{U_{x 2} U_{y 2} U_{s 3} U_{t 3}-U_{s 2} U_{t 2} U_{x 3} U_{y 3}}{U_{s 1} U_{t 1} U_{x 3} U_{y 3}-U_{s 3} U_{t 3} U_{x 1} U_{y 1}}\right|, \\
& \frac{m_{1}}{m_{3}}=\left|\frac{U_{x 3} U_{y 3} U_{s 2} U_{t 2}-U_{s 3} U_{t 3} U_{x 2} U_{y 2}}{U_{s 1} U_{t 1} U_{x 2} U_{y 2}-U_{s 2} U_{t 2} U_{x 1} U_{y 1}}\right|,
\end{aligned}
$$

and 
TABLE I. The mass ratios for each type of neutrino mass matrices up to first order in $s_{13}$.

\begin{tabular}{|c|c|}
\hline Type of texture & Mass ratios \\
\hline$A_{1}\left(M_{e e}=0 ; M_{e \mu}=0\right)$ & $\begin{array}{l}\frac{m_{1}}{m_{2}}=\tan ^{2} \theta_{12}\left(1-\frac{\cot \theta_{23}}{s_{12} c_{12}} s_{13} \cos \delta\right) \\
\frac{m_{1}}{m_{3}}=\tan \theta_{12} \tan \theta_{23} s_{13}\end{array}$ \\
\hline$A_{2}\left(M_{e e}=0 ; M_{e \tau}=0\right)$ & $\begin{array}{l}\frac{m_{1}}{m_{2}}=\tan ^{2} \theta_{12}\left(1+\frac{\tan \theta_{23}}{c_{12} s_{12}} s_{13} \cos \delta\right) \\
\frac{m_{1}}{m_{3}}=\tan \theta_{12} \cot \theta_{23} s_{13}\end{array}$ \\
\hline$B_{1}\left(M_{e \tau}=0 ; M_{\mu \mu}=0\right)$ & $\begin{array}{l}\frac{m_{1}}{m_{2}}=1+\frac{c_{23}}{c_{12} s_{12} s_{23}^{3}} s_{13} \cos \delta \\
\frac{m_{1}}{m_{3}}=\tan ^{2} \theta_{23}\left(1+\frac{\tan \theta_{23} \cot \theta_{12}}{s_{23}^{2}} s_{13} \cos \delta\right)\end{array}$ \\
\hline$B_{2}\left(M_{e \mu}=0 ; M_{\tau \tau}=0\right)$ & $\begin{array}{l}\frac{m_{1}}{m_{2}}=1-\frac{s_{23}}{c_{12} s_{12} c_{23}^{3}} s_{13} \cos \delta \\
\frac{m_{1}}{m_{3}}=\frac{1}{\tan ^{2} \theta_{23}}\left(1-\frac{\tan \theta_{23} \cot \theta_{12}}{c_{23}^{2}} s_{13} \cos \delta\right)\end{array}$ \\
\hline$B_{3}\left(M_{e \mu}=0 ; M_{\mu \mu}=0\right)$ & $\begin{array}{l}\frac{m_{1}}{m_{2}}=1-\frac{c_{23} \tan ^{2} \theta_{23}}{c_{12} s_{12} s_{23}^{3}} s_{13} \cos \delta \\
\frac{m_{1}}{m_{3}}=\tan ^{2} \theta_{23}\left(1-\frac{\cot \theta_{12}}{s_{23} c_{23}} s_{13} \cos \delta\right)\end{array}$ \\
\hline$B_{4}\left(M_{e \tau}=0 ; M_{\tau \tau}=0\right)$ & $\begin{array}{l}\frac{m_{1}}{m_{2}}=1+\frac{s_{23} \cot ^{2} \theta_{23}}{c_{12} s_{12} c_{23}^{3}} s_{13} \cos \delta \\
\frac{m_{1}}{m_{3}}=\frac{1}{\tan ^{2} \theta_{23}}\left(1+\frac{\cot \theta_{12}}{s_{23} c_{23}} s_{13} \cos \delta\right)\end{array}$ \\
\hline$C\left(M_{\mu \mu}=0 ; M_{\tau \tau}=0\right)$ & $\begin{array}{l}\frac{m_{1}}{m_{2}}=\frac{1}{\tan ^{2} \theta_{12}}\left(1-\frac{\tan \theta_{23}}{s_{12} c_{12}} s_{13} \cos \delta\right) \\
\frac{m_{1}}{m_{3}}=\frac{1}{\tan \theta_{12} \tan 2 \theta_{23} s_{13}} \\
\quad\left(1+\frac{4\left(-s_{12}^{2}+c_{12}^{2} \cos ^{2} 2 \theta_{23}\right)}{\sin 4 \theta_{22} \sin \theta_{12}} s_{13} \cos \delta\right)\end{array}$ \\
\hline
\end{tabular}

$\rho=-\frac{1}{2} \operatorname{Arg}\left(\frac{U_{x 2} U_{y 2} U_{s 3} U_{t 3}-U_{s 2} U_{t 2} U_{x 3} U_{y 3}}{U_{s 1} U_{t 1} U_{x 3} U_{y 3}-U_{s 3} U_{t 3} U_{x 1} U_{y 1}}\right)$, $\sigma=-\frac{1}{2} \operatorname{Arg}\left(\frac{U_{x 3} U_{y 3} U_{s 2} U_{t 2}-U_{s 3} U_{t 3} U_{x 2} U_{y 2}}{U_{s 1} U_{t 1} U_{x 2} U_{y 2}-U_{s 2} U_{t 2} U_{x 1} U_{y 1}}\right)-\delta$,

respectively. Using Eqs. (2), (6), and (7), we have shown these mass ratios up to first order in $s_{13}$ in Table I. These relations are useful while studying the phenomenology of two-texture zero neutrino mass matrices. In our numerical analysis, we have used Eqs. (6) and (7) to obtain the allowed parameter space. The mass matrices of the type $A_{1}, A_{2}, B_{1}$, $B_{2}, B_{3}, B_{4}$, and $C$ are found to be consistent with global data [16] on neutrino masses and mixing angles, and the cosmological bound on neutrino mass [17]. All the known parameters are randomly generated $\left(10^{7}\right.$ points $)$ in their $3 \sigma$ range with Gaussian distribution. Other parameters are generated with uniform distribution within their physical range. The best-fit point (bfp) and $1 \sigma$ range of these parameters are tabulated in Table II. We have also calculated the Jarlskog [18] $C P$ invariant $J_{C P}$ sensitive to Dirac phase $\delta$,

$$
J_{C P}=\operatorname{Im}\left\{V_{e 1} V_{\mu 2} V_{e 2}^{*} V_{\mu 1}^{*}\right\},
$$

and $s_{1}, s_{2} C P$ invariants $[19,20]$,

$$
\begin{aligned}
& s_{1}=\operatorname{Im}\left\{V_{e 1} V_{e 3}^{*}\right\}, \\
& s_{2}=\operatorname{Im}\left\{V_{e 2} V_{e 3}^{*}\right\},
\end{aligned}
$$

\begin{tabular}{|c|c|c|}
\hline \multirow[b]{2}{*}{ Type of texture } & \multicolumn{2}{|c|}{ bfp $\pm 1 \sigma$ in degrees $\left({ }^{\circ}\right)$} \\
\hline & Normal hierarchy & Inverted hierarchy \\
\hline$A_{1}$ & $\begin{array}{l}\theta_{12}=33.52_{-0.74}^{+0.74}, \theta_{13}=8.45_{-0.14}^{+0.14}, \theta_{23}=41.63_{-1.38}^{+1.38} \\
\rho=-77.42_{-34.71}^{+34.71}, \sigma=-102.60_{-48.37}^{+48.37}, \delta=67.58_{-31.99}^{+31.99}\end{array}$ & $\cdots$ \\
\hline$A_{2}$ & $\begin{array}{l}\theta_{12}=34.55_{-0.57}^{+0.57}, \theta_{13}=8.38_{-0.14}^{+0.14}, \theta_{23}=43.27_{-1.07}^{+1.07} \\
\rho=86.69_{-1.91}^{+1.91}, \sigma=-78.44_{-6.68}^{+6.68}, \delta=157.1_{-15.77}^{+15.77}\end{array}$ & $\cdots$ \\
\hline$B_{1}$ & $\begin{array}{l}\theta_{12}=33.75_{-0.57}^{+0.57}, \theta_{13}=8.45_{-0.14}^{+0.14}, \theta_{23}=41.10_{-1.07}^{+1.07} \\
\rho=-3.67_{-1.73}^{+1.73}, \sigma=-0.36_{-0.25}^{+0.25}, \delta=267.10_{-15.21}^{+15.21}\end{array}$ & $\begin{array}{l}\theta_{12}=33.52_{-0.77}^{+0.77}, \theta_{13}=8.47_{-0.13}^{+0.13}, \theta_{23}=45.76_{-0.52}^{+0.52}, \\
\rho=0.47_{-0.32}^{+0.32}, \sigma=-177.36_{-28.84}^{+28.84}, \delta=268.87_{-14.47}^{+14.47}\end{array}$ \\
\hline$B_{2}$ & $\begin{array}{l}\theta_{12}=33.60_{-0.76}^{+0.76}, \theta_{13}=8.45_{-0.14}^{+0.14}, \theta_{23}=45.75_{-0.47}^{+0.47} \\
\rho=0.46_{-0.50}^{+0.50}, \sigma=-174.92_{-42.41}^{+42.41}, \delta=266.91_{-21.29}^{+21.29}\end{array}$ & $\begin{array}{l}\theta_{12}=33.71_{-0.76}^{+0.76}, \theta_{13}=8.45_{-0.14}^{+0.14}, \theta_{23}=41.03_{-1.26}^{+1.26} \\
\rho=-1.85_{-0.55}^{+0.55}, \sigma=-1.45_{-0.45}^{+0.45}, \delta=269.00_{-13.24}^{+13.24}\end{array}$ \\
\hline$B_{3}$ & $\begin{array}{l}\theta_{12}=33.64_{-0.74}^{+0.74}, \theta_{13}=8.49_{-0.14}^{+0.14}, \theta_{23}=41.10_{-1.30}^{+1.30} \\
\rho=2.43_{-0.97}^{+0.97}, \sigma=-177.50_{-31.89}^{+31.89}, \delta=267.80_{-16.31}^{+16.31}\end{array}$ & $\begin{array}{l}\theta_{12}=33.58_{-0.75}^{+0.75}, \theta_{13}=8.47_{-0.14}^{+0.14}, \theta_{23}=45.78_{-0.52}^{+0.52} \\
\rho=-0.47_{-0.32}^{+0.32}, \sigma=-0.31_{-0.18}^{+0.18}, \delta=269.47_{-9.20}^{+9.20}\end{array}$ \\
\hline$B_{4}$ & $\begin{array}{l}\theta_{12}=33.66_{-0.71}^{+0.71}, \theta_{13}=8.45_{-0.15}^{+0.15}, \theta_{23}=45.87_{-0.54}^{+0.54}, \\
\rho=-0.47_{-0.34}^{+0.34}, \sigma=-0.25_{-0.77}^{+0.77}, \delta=268.50_{-10.90}^{+10.90}\end{array}$ & $\begin{array}{l}\theta_{12}=33.74_{-0.74}^{+0.74}, \theta_{13}=8.45_{-0.14}^{+0.14}, \theta_{23}=41.29_{-1.27}^{+1.27} \\
\rho=2.24_{-0.90}^{+0.90}, \sigma=-167.4_{-30.86}^{+30.86}, \delta=266.82_{-15.60}^{+15.60}\end{array}$ \\
\hline$C$ & $\cdots$ & $\begin{array}{l}\theta_{12}=33.54_{-0.75}^{+0.75}, \theta_{13}=8.44_{-0.14}^{+0.14}, \theta_{23}=41.08_{-1.26}^{+1.26} \\
\rho=46.65_{-12.33}^{+12.33}, \sigma=-165.06_{-19.07}^{+19.07}, \delta=292.80_{-15.17}^{+15.17}\end{array}$ \\
\hline
\end{tabular}

sensitive to Majorana phases $\rho$ and $\sigma$ (Table III).

TABLE II. The neutrino mixing parameters in two-texture zero neutrino mass matrices. 
TABLE III. The $C P$ invariants in two-texture zero neutrino mass matrices.

\begin{tabular}{lcccc}
\hline \hline Type of texture & Hierarchy & $J_{C P}(\mathrm{bfp} \pm 1 \sigma)$ & $s_{1}(\mathrm{bfp} \pm 1 \sigma)$ & $s_{2}(\mathrm{bfp} \pm 1 \sigma)$ \\
\hline$A_{1}$ & $\mathrm{NH}$ & $(2.67 \pm 1.16) \times 10^{-2}$ & $(-0.10 \pm 0.04) \times 10^{-2}$ & $(-0.14 \pm 0.06) \times 10^{-2}$ \\
$A_{2}$ & $\mathrm{NH}$ & $(1.60 \pm 0.80) \times 10^{-2}$ & $(-0.28 \pm 0.03) \times 10^{-2}$ & $(0.40 \pm 0.03) \times 10^{-2}$ \\
$B_{1}$ & $\mathrm{NH}$ & $(3.21 \pm 0.63) \times 10^{-2}$ & $(-1.38 \pm 0.98) \times 10^{-5}$ & $(-8.30 \pm 3.60) \times 10^{-5}$ \\
& $\mathrm{IH}$ & $(3.22 \pm 0.85) \times 10^{-2}$ & $(0.64 \pm 0.17) \times 10^{-2}$ & $(0.43 \pm 0.11) \times 10^{-2}$ \\
$B_{2}$ & $\mathrm{NH}$ & $(3.29 \pm 0.34) \times 10^{-2}$ & $(-0.66 \pm 0.06) \times 10^{-2}$ & $(0.44 \pm 0.04) \times 10^{-2}$ \\
& $\mathrm{IH}$ & $(3.22 \pm 0.62) \times 10^{-2}$ & $(-4.97 \pm 1.79) \times 10^{-5}$ & $(-1.43 \pm 0.44) \times 10^{-5}$ \\
$B_{3}$ & $\mathrm{NH}$ & $(3.22 \pm 0.58) \times 10^{-2}$ & $(-6.54 \pm 1.19) \times 10^{-3}$ & $(4.41 \pm 0.80) \times 10^{-3}$ \\
& $\mathrm{IH}$ & $(3.29 \pm 0.68) \times 10^{-2}$ & $(-1.65 \pm 0.65) \times 10^{-4}$ & $(-1.01 \pm 0.65) \times 10^{-4}$ \\
$B_{4}$ & $\mathrm{NH}$ & $(3.32 \pm 0.53) \times 10^{-2}$ & $(-1.99 \pm 0.94) \times 10^{-6}$ & $(-1.20 \pm 0.87) \times 10^{-5}$ \\
& $\mathrm{IH}$ & $(3.23 \pm 0.55) \times 10^{-2}$ & $(-6.50 \pm 1.10) \times 10^{-3}$ & $(4.30 \pm 0.75) \times 10^{-3}$ \\
$C$ & $\mathrm{IH}$ & $(2.95 \pm 0.38) \times 10^{-2}$ & $(-6.19 \pm 0.64) \times 10^{-3}$ & $(5.25 \pm 0.59) \times 10^{-3}$ \\
\hline \hline
\end{tabular}

\section{MAJORANA UNITARITY TRIANGLES IN TWO-TEXTURE ZERO MODEL}

In Majorana triangles [Eq. (4)], all the terms are rephasing invariant and hence do not rotate in the complex plane under rephasing transformations. The study of these triangles is of great physical significance due to their dependence on Majorana phases [21,22]. The MTs $\Delta_{12}$

TABLE IV. The relation between Majorana phases and angles of the Majorana unitarity triangle.

\begin{tabular}{|c|c|}
\hline $\begin{array}{l}\text { Type of } \\
\text { texture }\end{array}$ & $\begin{array}{l}\text { Majorana phases in terms of angles } \\
\text { of the Majorana unitarity triangle }\end{array}$ \\
\hline$A_{1}$ & $\begin{array}{c}\rho=-\frac{1}{2}\left(\gamma_{12}-\pi+\operatorname{Arg}\left(\frac{U_{e 3} U_{\mu 2} U_{\mu 1} U_{e 2}^{2}-U_{e 2}^{3} U_{\mu 3} U_{\mu 1}}{U_{\mu 2} U_{e 1}^{3} U_{\mu 3}-U_{\mu 2} U_{e 3} U_{\mu 1} U_{e 1}^{2}}\right)\right) \\
\sigma=-\frac{1}{2}\left(\gamma_{12}-\pi+\operatorname{Arg}\left(\frac{U_{e 2}^{3} U_{e 3} U_{\mu 3} U_{\mu 1}-U_{e 3}^{2} U_{e 2}^{2} U_{\mu 2} U_{\mu 1}}{U_{e 1}^{3} U_{e 2} U_{\mu 2}^{2}-U_{e 2}^{2} U_{e 1}^{2} U_{\mu 1} U_{\mu 2}}\right)\right)-\delta\end{array}$ \\
\hline$A_{2}$ & $\begin{array}{c}\rho=\frac{1}{2}\left(\beta_{12}-\pi+\operatorname{Arg}\left(\frac{U_{e 3} U_{\tau 2}^{2} U_{\tau 1}-U_{\tau 3} U_{e 2} U_{\tau 1} U_{\tau 2}}{U_{e 1} U_{\tau 1} U_{\tau 2} U_{\tau 3}-U_{\tau 1}^{2} U_{e 3} U_{\tau 2}}\right)\right), \\
\sigma=-\frac{1}{2}\left(\beta_{12}-\pi+\operatorname{Arg}\left(\frac{U_{e 2}^{3} U_{e 3} U_{\tau 3}-U_{e 3}^{2} U_{e 2}^{2} U_{\tau 2}}{U_{e 1} U_{e 2} U_{\tau 2}^{2} U_{\tau 1}-U_{e 2}^{2} U_{\tau 1}^{2} U_{\tau 2}}\right)\right)-\delta .\end{array}$ \\
\hline$B_{1}$ & $\begin{array}{c}\rho=-\frac{1}{2}\left(\beta_{23}-\pi+\operatorname{Arg}\left(\frac{U_{\mu 2}^{2} U_{e 3} U_{\tau 3}^{2} U_{e 2}-U_{\mu 3}^{2} U_{e 2}^{2} U_{\tau 2} U_{\tau 3}}{U_{\mu 3}^{2} U_{e 1} U_{\tau 1} U_{\tau 2} U_{e 3}-U_{\mu 1}^{2} U_{e 3}^{2} U_{\tau 2} U_{\tau 3}}\right)\right), \\
\sigma=-\frac{1}{2}\left(\beta_{23}-\pi+\operatorname{Arg}\left(\frac{U_{\mu 3}^{2} U_{e 2}^{2} U_{\tau 2} U_{\tau 3}-U_{\mu 2}^{2} U_{e 2} U_{e 3} U_{\tau 3}^{2}}{U_{\mu 2}^{2} U_{e 1} U_{\tau 1} U_{\tau 2} U_{e 3}-U_{\mu 1}^{2} U_{e 2} U_{\tau 2}^{2} U_{e 3}}\right)\right)-\delta .\end{array}$ \\
\hline$B_{2}$ & $\begin{array}{c}\rho=-\frac{1}{2}\left(\gamma_{23}-\pi+\operatorname{Arg}\left(\frac{U_{\tau 2}^{2} U_{e 3}^{2} U_{\mu 3} U_{\mu 2}-U_{\tau 3}^{2} U_{e 2} U_{\mu 2}^{2} U_{e 3}}{U_{\tau 3}^{2} U_{e 1} U_{\mu 1} U_{e 2} U_{\mu 3}-U_{\tau 1}^{2} U_{e 3} U_{\mu 3}^{2} U_{e 2}}\right)\right), \\
\sigma=-\frac{1}{2}\left(\gamma_{23}-\pi+\operatorname{Arg}\left(\frac{U_{\tau 3}^{2} U_{e 2} U_{\mu 2}^{2} U_{e 3}-U_{\tau 2}^{2} U_{e 3}^{2} U_{\mu 2} U_{\mu 3}}{U_{\tau 2}^{2} U_{e 1} U_{\mu 1} U_{e 2} U_{\mu 3}-U_{\tau 1}^{2} U_{e 2}^{2} U_{\mu 2} U_{\mu 3}}\right)\right)-\delta .\end{array}$ \\
\hline$B_{3}$ & $\begin{array}{c}\rho=-\frac{1}{2}\left(\gamma_{31}-\pi+\operatorname{Arg}\left(\frac{U_{\mu 2}^{2} U_{e 3} U_{\mu 3}^{2} U_{e 1}-U_{e 2} U_{\mu 2} U_{\mu 3}^{3} U_{e 1}}{U_{\mu 3}^{2} U_{e 1} U_{\mu 1}^{2} U_{e 3}-U_{e 3}^{2} U_{\mu 3} U_{\mu 1}^{3}}\right)\right), \\
\sigma=-\frac{1}{2}\left(\gamma_{31}-\pi+\operatorname{Arg}\left(\frac{U_{\mu 3}^{2} U_{e 2} U_{\mu 2} U_{e 1}-U_{\mu 2}^{2} U_{e 3} U_{\mu 3}^{2} U_{e 1}}{U_{\mu 2}^{2} U_{e 1} U_{\mu 1} U_{e 3}-U_{e 2} U_{\mu 2} U_{\mu 1}^{3} U_{e 3}}\right)\right)-\delta .\end{array}$ \\
\hline$B_{4}$ & $\begin{array}{l}\rho=-\frac{1}{2}\left(\beta_{31}-\pi+\operatorname{Arg}\left(\frac{U_{\tau 2}^{2} U_{e 3}^{2} U_{\tau 3} U_{\tau 1}-U_{e 2} U_{\tau 2} U_{\tau 3}^{2} U_{e 3} U_{\tau 1}}{U_{\tau 3}^{3} U_{e 1}^{2} U_{\tau 1} U_{\tau 3}-U_{e 3} U_{\tau 3}^{2} U_{\tau 1}^{2} U_{e 1}}\right)\right), \\
\sigma=\frac{1}{2}\left(\beta_{31}-\pi+\operatorname{Arg}\left(\frac{U_{\tau 3}^{2} U_{e 2} U_{\tau 2} U_{\tau 1} U_{e 3}-U_{\tau 2}^{2} U_{\tau 1} U_{e 3}^{2} U_{\tau 3}}{U_{\tau 2}^{2} U_{e 1}^{2} U_{\tau 1} U_{\tau 3}-U_{e 2} U_{\tau 2} U_{\tau 1}^{2} U_{\tau 3} U_{e 1}}\right)\right)-\delta .\end{array}$ \\
\hline$C$ & $\begin{array}{c}\rho=\frac{1}{2}\left(\alpha_{31}-\pi+\operatorname{Arg}\left(\frac{U_{\tau 2}^{2} U_{\mu 3}^{3} U_{\tau 3}^{3}-U_{\mu 2}^{2} U_{\tau 3}^{5} U_{\mu 3}}{U_{\mu 1}^{3} U_{\tau 3}^{2} U_{\tau 1}^{3}-U_{\mu 3}^{2} U_{\tau 1}^{5} U_{\mu 1}}\right)\right), \\
\sigma=\frac{1}{2}\left(\alpha_{31}-\pi+\operatorname{Arg}\left(\frac{U_{\tau 3}^{3} U_{\mu 1} U_{\tau 2}^{2}-U_{\mu 3}^{2} U_{\tau 2}^{2} U_{\mu 1} U_{\tau 3}}{U_{\mu 1}^{2} U_{\tau 2}^{2} U_{\mu 3} U_{\tau 1}-U_{\mu 2}^{2} U_{\tau 1}^{3} U_{\mu 3}}\right)\right)-\delta .\end{array}$ \\
\hline
\end{tabular}

and $\Delta_{31}$ are sensitive to $\rho$ and $\sigma$, respectively, whereas $\Delta_{23}$ is sensitive to both Majorana phases $(\rho, \sigma)$. In general, the sides and angles of the MT can be expressed as

$$
\begin{gathered}
\left(S_{1}, S_{2}, S_{3}\right)=\left(\left|V_{e f} V_{e f^{\prime}}\right|,\left|V_{\mu f} V_{\mu f^{\prime}}\right|,\left|V_{\tau f} V_{\tau f^{\prime}}\right|\right), \\
\alpha_{f f^{\prime}}=\operatorname{Arg}\left(-\frac{V_{\mu f} V_{\mu f^{\prime}}^{*}}{V_{\tau f} V_{\tau f^{\prime}}^{*}}\right), \quad \beta_{f f^{\prime}}=\operatorname{Arg}\left(-\frac{V_{\tau f} V_{\tau f^{\prime}}^{*}}{V_{e f} V_{e f^{\prime}}^{*}}\right), \\
\gamma_{f f^{\prime}}=\operatorname{Arg}\left(-\frac{V_{e f} V_{e f^{\prime}}^{*}}{V_{\mu f} V_{\mu f^{\prime}}^{*}}\right),
\end{gathered}
$$

where $S_{1}, S_{2}, S_{3}$ are three sides and $\alpha, \beta, \gamma$ are three angles with subscript $f, f^{\prime}=(1,2,3)$ and $f \neq f^{\prime}$. We have obtained a relation between Majorana phases and interior angles of the MT for class $A, B$, and $C$-type neutrino mass matrices, which are tabulated in Table IV. As can be seen from the Table IV, the Majorana phases depend only on one of the interior angles of Majorana triangles in the twotexture zero neutrino mass model. These relations are useful to identify the corresponding Majorana triangle for each type of two-texture zero neutrino mass matrix. Hence, we choose the Majorana triangle $\Delta_{12}$ for $A_{1}$ and $A_{2}$, $\Delta_{23}$ for $B_{1}$ and $B_{2}, \Delta_{31}$ for $B_{3}, B_{4}$, and $C$.

We have also constructed the Majorana triangles for each type of two-texture zero neutrino mass matrix (Figs. 1-3) with best-fit values of neutrino mixing parameters shown in Table II.

\section{STATUS OF NEUTRINOLESS DOUBLE BETA DECAY $(0 \nu \beta \beta)$ IN THE TWO-TEXTURE ZERO NEUTRINO MASS MODEL}

The sensitivities of current and future experiments to LNV processes such as $K^{+} \rightarrow \mu^{+} \mu^{+} \pi^{-}$decay [23-25], the nuclear muon to positron [26,27], trimuonium production in neutrino muon scattering [28], the process $e^{+} p \rightarrow$ $\bar{\nu} l_{1}^{+} l_{2}^{+} X$ [29], and $0 \nu \beta \beta$ decay [30-32] have been extensively studied in the literature, and the existence of these will demonstrate the Majorana nature of the neutrinos. The 

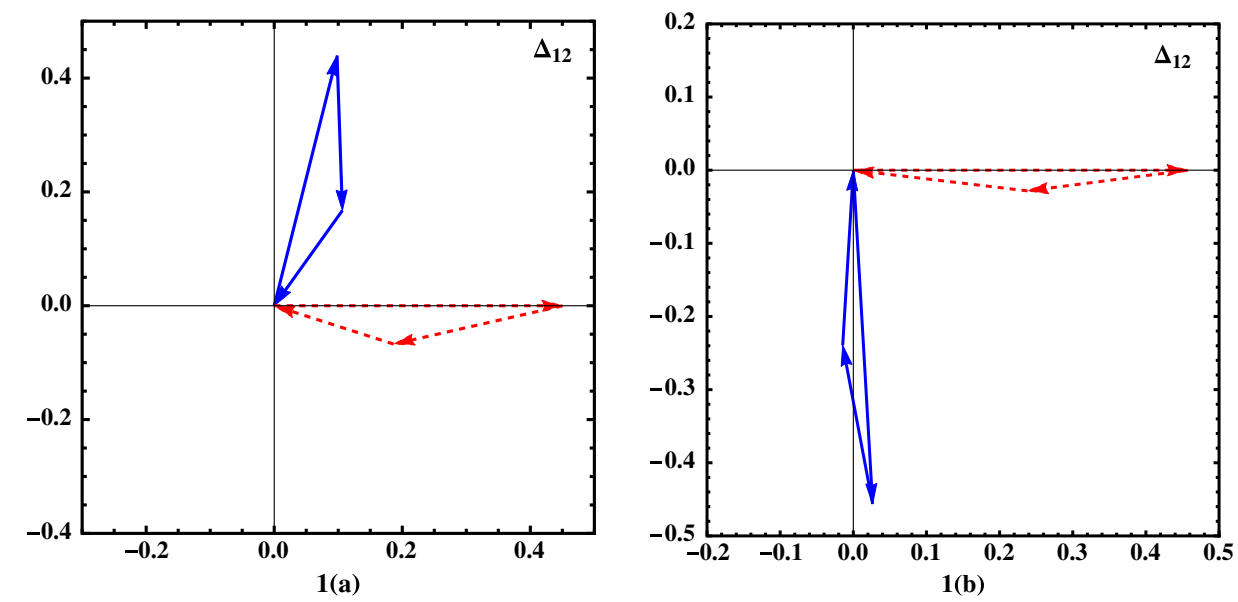

FIG. 1. Majorana triangles for class $A$ neutrino mass matrices with normal hierarchical (NH) neutrino masses, MT for type $A_{1}\left(A_{2}\right)$ with $\mathrm{NH}$ in the left (right).

sensitivities of the current experiments searching for these processes are much less in comparison to experimental sensitivity to the $0 \nu \beta \beta$ decay process. $0 \nu \beta \beta$ decay is the most promising probe of observing lepton number violation and will shed light on the mechanism of neutrino mass generation. The effective Majorana mass, $\langle m\rangle_{e e}$, may vanish for normal hierarchical neutrino masses; however, for inverted hierarchical neutrino masses there exists a lower bound on $\langle m\rangle_{e e}$ providing bright prospects for observation of $0 \nu \beta \beta$ decay. Although it will be difficult to measure Majorana phases in these experiments, a correlation between them can always be obtained. One can uniquely determine both Majorana phases for the case of vanishing $\langle m\rangle_{e e}$ [33]. The decay width of the $0 \nu \beta \beta$ is proportional to the effective Majorana mass, $\langle m\rangle_{e e}$, given by [34]

$\langle m\rangle_{e e} \equiv \sum_{i=1}^{3} V_{e i}^{2} m_{i},=m_{1}\left|U_{e 1}^{2}\right|+m_{2}\left|U_{e 2}^{2}\right| e^{2 i \rho}+m_{3}\left|U_{e 3}^{2}\right| e^{2 i \sigma}$.

Using the allowed parameter space obtained for twotexture zero neutrino mass matrices shown in Table II, we have obtained the $1 \sigma$ range of effective Majorana mass, $\left|\langle m\rangle_{e e}\right|$, for each type of two-texture zero neutrino mass model that has been tabulated in Table V. For class A-type neutrino mass models, the effective Majorana mass, $\langle m\rangle_{e e}$, is vanishing; thus observation of $0 \nu \beta \beta$ decay rules out these classes of neutrino mass models. The sensitivities of the $0 \nu \beta \beta$ experiments like KamLAND-ZEN [35,36], GERDA [37], CUORE [38,39], NEXT [40,41], and EXO-200 $[42,43]$ have set strong bounds on effective Majorana mass, $\left|\langle m\rangle_{e e}\right|$, tabulated in Table VI. Recently, the strongest constraint has been obtained by KamLAND-ZEN [36]. The predictions for effective Majorana mass, $\left|\langle m\rangle_{e e}\right|$, for class $B$ and $C$ are found to be well within the sensitivity reach of current and future $0 \nu \beta \beta$ decay experiments (Table VI). However, nonobservation of $0 \nu \beta \beta$ decay favors class $A$ and class $B$ (with $\mathrm{NH}$ ) type neutrino mass models.

\section{OBSERVABLES SENSITIVE TO GEOMETRIC PARAMETERS OF THE MAJORANA UNITARITY TRIANGLE}

In the case that the massive neutrinos are identified as Majorana particles, the next inevitable question will arise regarding the determination of Majorana $C P$ violating phases relevant to future neutrino experiments. This question can be answered on the basis of various LNV processes (discussed in an earlier section) that are sensitive to Majorana nature of neutrino masses. One such LNV process is neutrino to antineutrino oscillations. The $C P$ asymmetry $\left(\Delta P_{C P}\right)$ in neutrino-antineutrino oscillations shows sensitivity to all three $C P$ violating phases of matrix $V$ and can be written as $[45,46]$

$$
\begin{aligned}
& P_{\nu_{l} \rightarrow \bar{\nu}_{l^{\prime}}}=\frac{|\mathcal{K}|^{2}}{E^{2}}\left(\left|m_{l l^{\prime}}\right|^{2}-4 \sum_{f<f^{\prime}} m_{f} m_{f^{\prime}} \Re\left(V_{l f} V_{l^{\prime} f} V_{l f^{\prime}}^{*} V_{l^{\prime} f^{\prime}}^{*}\right) \sin ^{2} \frac{\Delta m_{f^{\prime} f}^{2} L}{4 E}+2 \sum_{f<f^{\prime}} m_{f} m_{f^{\prime}} \Im\left(V_{l f} V_{l^{\prime} f} V_{l f^{\prime}}^{*} V_{l^{\prime} f^{\prime}}^{*}\right) \sin \frac{\Delta m_{f^{\prime} f}^{2} L}{2 E}\right), \\
& P_{\bar{\nu}_{l} \rightarrow \nu_{l^{\prime}}}=\frac{|\overline{\mathcal{K}}|^{2}}{E^{2}}\left(\left|m_{l l^{\prime}}\right|^{2}-4 \sum_{f<f^{\prime}} m_{f} m_{f^{\prime}} \Re\left(V_{l f} V_{l^{\prime} f} V_{l f^{\prime}}^{*} V_{l^{\prime} f^{\prime}}^{*}\right) \sin ^{2} \frac{\Delta m_{f^{\prime} f}^{2} L}{4 E}-2 \sum_{f<f^{\prime}} m_{f} m_{f^{\prime}} \Im\left(V_{l f} V_{l^{\prime} f} V_{l f^{\prime}}^{*} V_{l^{\prime} f^{\prime}}^{*}\right) \sin \frac{\Delta m_{f^{\prime} f}^{2} L}{2 E}\right),
\end{aligned}
$$

and 

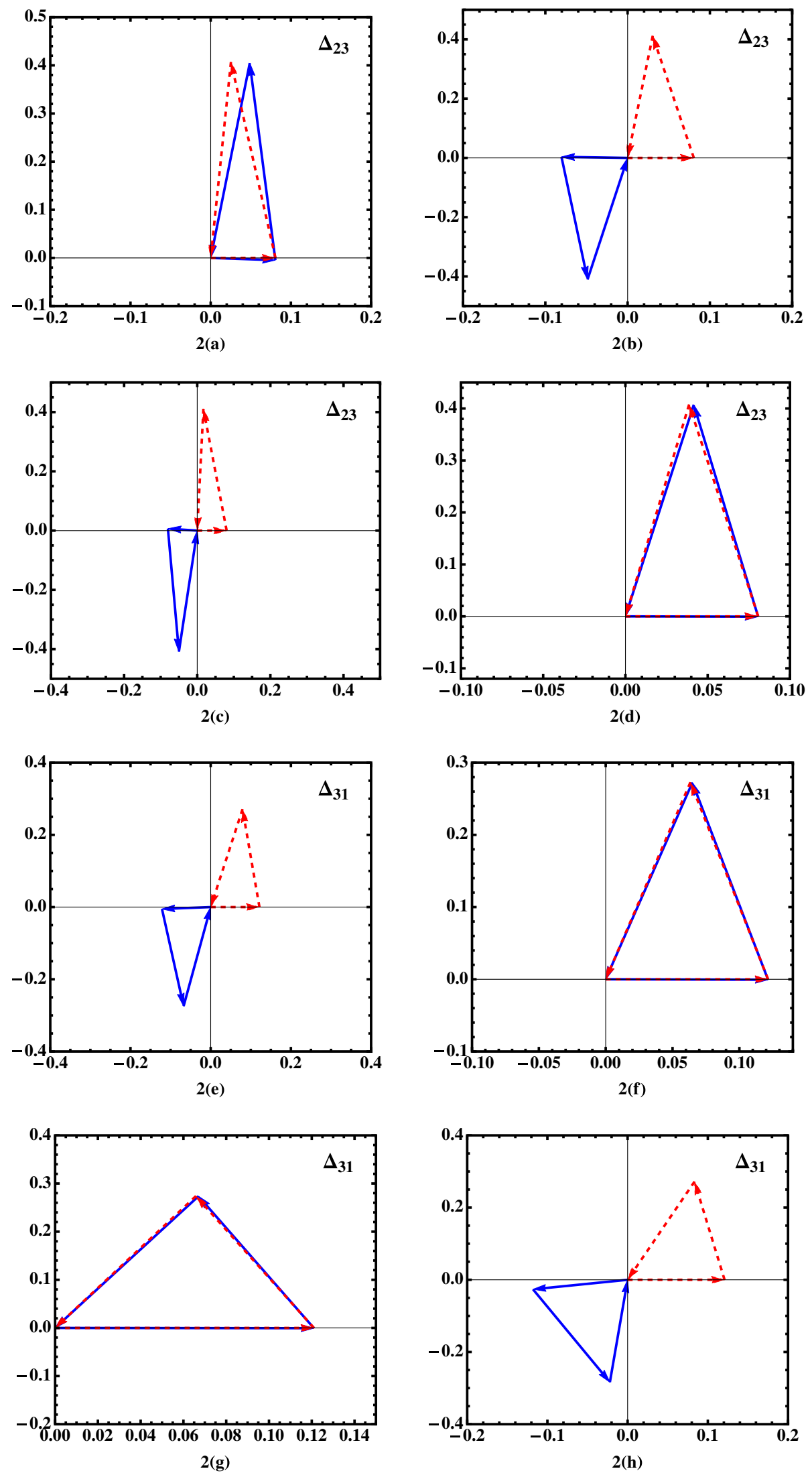

FIG. 2. Majorana triangles for class $B$ neutrino mass matrices with normal and inverted hierarchical (IH) neutrino masses, MT for $B_{1}$, $B_{2}, B_{3}$, and $B_{4}$ with $\mathrm{NH}$ (left) and $\mathrm{IH}$ (right). 


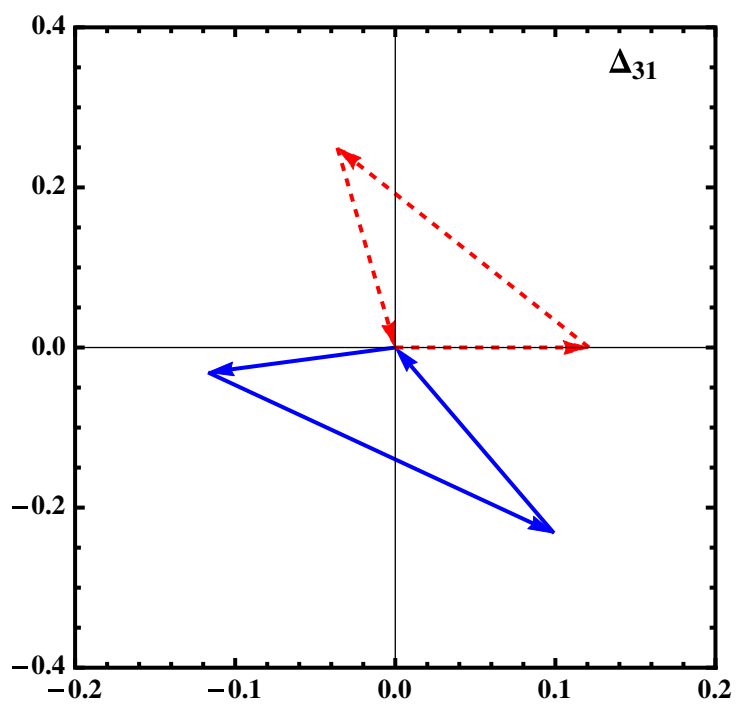

FIG. 3. Majorana triangles for class $C$ neutrino mass matrices with inverted hierarchical neutrino masses.

$\Delta P_{C P}=4 \frac{|\mathcal{K}|^{2}}{E^{2}} \sum_{f<f^{\prime}} m_{f} m_{f^{\prime}} \Im\left(V_{l f} V_{l^{\prime} f} V_{l f^{\prime}}^{*} V_{l^{\prime} f^{\prime}}^{*}\right) \sin \frac{\Delta m_{f^{\prime} f}^{2} L}{2 E}$,

where $\Delta m_{f^{\prime} f}^{2} \equiv m_{f^{\prime}}^{2}-m_{f}^{2}, \mathcal{K}$ and $\overline{\mathcal{K}}$ are kinematical factors satisfying $|\mathcal{K}|=|\overline{\mathcal{K}}|, E$ is the neutrino (antineutrino) energy $(\mathrm{GeV})$, and $L$ is baseline $(\mathrm{km})$. The mass term $m_{l l^{\prime}} \equiv \sum_{f} m_{f} V_{l f} V_{l^{\prime} f}$ for $l, l^{\prime}=e, \mu, \tau$, which is $\left(l, l^{\prime}\right)$ element of Majorana neutrino mass matrix $M$. The above equations depend on the product of two quantities $V_{l f} V_{l f^{\prime}}^{*}$ and $V_{l^{\prime} f} V_{l^{\prime} f^{\prime}}^{*}$ from which one can deduce two sides and one angle of the Majorana unitarity triangle using Eqs. (13) and (14). For example, in the case of $A_{1}$-type neutrino mass matrices we can obtain the angle $\gamma_{12}$ of $\Delta_{12}$ MT (thus, Majorana phases $\rho$ and $\sigma)$ from neutrino-antineutrino oscillation probabilities. Similarly, we can determine the Majorana $C P$ phases for all classes of the two-texture zero model, which can, in principle, be probed in neutrino-antineutrino oscillations.

TABLE V. $\left|\langle m\rangle_{e e}\right|(\mathrm{eV})$ for each type of two-texture zero neutrino mass matrix.

\begin{tabular}{llc}
\hline \hline Texture & $\mathrm{NH}(\mathrm{bfp} \pm 1 \sigma)$ & $\mathrm{IH}(\mathrm{bfp} \pm 1 \sigma)$ \\
\hline$A_{1}$ & $\left|\langle m\rangle_{e e}\right|=0$ & $\ldots$ \\
$A_{2}$ & $\left|\langle m\rangle_{e e}\right|=0$ & $\ldots$ \\
$B_{1}$ & $\left|\langle m\rangle_{e e}\right|=0.059 \pm 0.016$ & $\left|\langle m\rangle_{e e}\right|=0.181 \pm 0.066$ \\
$B_{2}$ & $\left|\langle m\rangle_{e e}\right|=0.173 \pm 0.088$ & $\left|\langle m\rangle_{e e}\right|=0.080 \pm 0.014$ \\
$B_{3}$ & $\left|\langle m\rangle_{e e}\right|=0.061 \pm 0.016$ & $\left|\langle m\rangle_{e e}\right|=0.033 \pm 0.015$ \\
$B_{4}$ & $\left|\langle m\rangle_{e e}\right|=0.180 \pm 0.113$ & $\left|\langle m\rangle_{e e}\right|=0.084 \pm 0.016$ \\
$C$ & $\cdots$ & $\left|\langle m\rangle_{e e}\right|=0.065 \pm 0.043$ \\
\hline \hline
\end{tabular}

\section{RESULTS AND DISCUSSION}

In class $A, \cos \delta$ should be positive (negative) for $A_{1}\left(A_{2}\right)$ because $\frac{m_{1}}{m_{2}}<1$. So, $\delta$ can be in the I or IV quadrant for $A_{1}$ and in II and III quadrant for the $A_{2}$-type mass matrix. These theoretical predictions are consistent with the obtained best-fit values of $\delta$ shown in Table II.

Class $B$ yields a quasidegenerate spectrum of neutrino masses. For mass ratio $\frac{m_{1}}{m_{2}}<1, \cos \delta$ should be negative (positive) for $B_{1}$ and $B_{4}\left(B_{2}\right.$ and $\left.B_{3}\right)$. We find that $\delta=267.10^{\circ}, 266.91^{\circ}, 267.80^{\circ}, 268.50^{\circ}\left(268.87^{\circ}, 269.00^{\circ}\right.$, $269.47^{\circ}, 266.82^{\circ}$ ) for $B_{1}, B_{2}, B_{3}$, and $B_{4}$ with $\mathrm{NH}(\mathrm{IH})$, respectively. The important point here is to note that the best-fit values of $\delta$ come out to be nearly equal to $\frac{3 \pi}{2}$ for class $\mathrm{B}$, which is in accordance with the experimental value of $\delta$ predicted by the combined analysis of T2K and Daya Bay experiments [47].

In class $C$, for $\frac{m_{1}}{m_{2}}<1$, the factor $\tan 2 \theta_{23} \cos \delta$ should be positive and $\delta$ must lie either in the I or IV quadrant. This prediction is found to be consistent with the best-fit value of $\delta=292.80^{\circ}$ (Table II). In Table III, we have obtained the $C P$ invariants corresponding to Dirac and Majorana-type $C P$ violation for class $A, B$, and $C$ neutrino mass matrices. The existence of nonzero $C P$ violation is a generic prediction of two-texture zero neutrino mass models. In Table IV, we have also obtained the expressions for Majorana phases in terms of the interior angle of Majorana unitarity triangle for class $A, B$, and $C$. We observe that the Majorana phases depend only on one independent geometric parameter of MT for each class of two-texture zero neutrino mass model. These relations are imperative while testing the two-texture zero neutrino mass model in LNV processes such as neutrinoantineutrino oscillations. The orientation of these triangles depends on the Majorana phases as

$$
\begin{aligned}
& \Delta_{12} \equiv e^{i \rho}\left(U_{e 1} U_{e 2}^{*}+U_{\mu 1} U_{\mu 2}^{*}+U_{\tau 1} U_{\tau 2}^{*}\right), \\
& \Delta_{23} \equiv e^{i(\delta+\sigma-\rho)}\left(U_{e 2} U_{e 3}^{*}+U_{\mu 2} U_{\mu 3}^{*}+U_{\tau 2} U_{\tau 3}^{*}\right), \\
& \Delta_{31} \equiv e^{i(\delta+\sigma)}\left(U_{e 3} U_{e 1}^{*}+U_{\mu 3} U_{\mu 1}^{*}+U_{\tau 3} U_{\tau 1}^{*}\right) .
\end{aligned}
$$

TABLE VI. Sensitivities to effective Majorana mass, $\left|\langle m\rangle_{e e}\right|$, of various $0 \nu \beta \beta$ decay experiments [44].

\begin{tabular}{ll}
\hline \hline Experiment & $\left|\langle m\rangle_{e e}\right|(\mathrm{eV})$ \\
\hline EXO-200 (4 yr) & $0.075-0.2$ \\
nEXO (5 yr) & $0.012-0.029$ \\
nEXO (5 yr + 5 yr w/Ba tagging) & $0.005-0.011$ \\
KamLAND-ZEN (300 kg, 3 yr) & $0.045-0.11$ \\
GERDA phase II & $0.09-0.29$ \\
CUORE (5 yr) & $0.051-0.133$ \\
SNO+ & $0.07-0.14$ \\
SuperNEMO & $0.05-0.15$ \\
NEXT & $0.03-0.1$ \\
MAJORANA demonstrator & $0.06-0.17$ \\
\hline \hline
\end{tabular}


Using Eq. (19) along with values of mixing parameters as shown in Table II, the Majorana triangles have been constructed as shown with solid lines in Figs. 1-3. For reference, the dashed triangles in Figs. 1-3 are obtained assuming $\rho$ and $\sigma$ equal to 0 . In general, we find that the Majorana triangles provide an indubitable signal towards nonzero $C P$ violation in this class of models because none of the sides of the triangle is parallel to the axis and all the Majorana triangles having nonvanishing area.

\section{CONCLUSIONS}

In conclusion, we have investigated $C P$ violation in neutrino mass models with two-texture zeros. In particular, we have obtained a possible connection between Majorana phases $(\rho, \sigma)$ and independent geometric parameters of the MT. We find that Majorana phases depend on one interior angle of the MT, i.e., $\gamma_{12}$ in $A_{1}, \beta_{12}$ in $A_{2}, \beta_{23}$ in $B_{1}, \gamma_{23}$ in $B_{2}, \gamma_{31}$ in $B_{3}, \beta_{31}$ in $B_{4}$ and $\alpha_{31}$ in the class $C$-type neutrino mass matrix. This analysis is important in light of the future neutrino oscillation experiments focusing on measuring Dirac-type $C P$ violation phase $\delta$. Also, these relations are imperative while testing the two-texture zero neutrino mass model in LNV processes such as neutrino-antineutrino oscillations. We have also obtained the best-fit and $\pm 1 \sigma$ values of neutrino oscillation parameters and $C P$ rephasing invariants for class $A, B$, and $C$ neutrino mass matrices. We find that for class $B$, the best-fit value lies very close to $\delta \approx \frac{3 \pi}{2}$, which is in accordance with T2K and Daya Bay experiments [47]. The nonzero area and nontrivial orientation of Majorana triangles shows that two-texture zero neutrino mass matrices are necessarily $C P$ violating.

The current and future $0 \nu \beta \beta$ decay experiments can shed light on two-texture zero neutrino mass models. As long as the fixing of parameters (absolute neutrino mass and Majorana phases) is concerned, the nonobservation of $0 \nu \beta \beta$ decay is more predictive than the situation in which $0 \nu \beta \beta$ decay is observed. The predictions for effective Majorana mass, $\left|\langle m\rangle_{e e}\right|$, for class $B$ and $C$ are found to be well within the sensitivity reach of current and future $0 \nu \beta \beta$ decay experiments (Table VI). The nonobservation of $0 \nu \beta \beta$ decay favors class $A$ and class $B$ (with $\mathrm{NH}$ ) type twotexture zero neutrino mass matrices while class $C$ is ruled out.

\section{ACKNOWLEDGMENTS}

S. V. acknowledges the financial support provided by University Grants Commission (UGC)-Basic Science Research (BSR), Government of India vide Grant No. F.20-2(03)/2013(BSR). S. B. acknowledges the financial support provided by the Central University of Himachal Pradesh. The authors also acknowledge the Department of Physics and Astronomical Science for providing necessary facilities to carry out this work.
[1] M. P. Decowski, Nucl. Phys. B908, 52 (2016).

[2] M. H. Ahn et al., Phys. Rev. D 74, 072003 (2006).

[3] S. N. Ahmed et al., Phys. Rev. Lett. 92, 181301 (2004).

[4] J. Hosaka et al., Phys. Rev. D 74, 032002 (2006).

[5] F. An et al., Phys. Rev. Lett. 108, 171803 (2012).

[6] S. M. Bilenky, J. Hosek, and S. T. Petcov, Phys. Lett. 94B, 495 (1980).

[7] G. C. Branco and M. N. Rebelo, Phys. Rev. D 79, 013001 (2009).

[8] P. H. Frampton, S. L. Glashow, and D. Marfatia, Phys. Lett. B 536, 79 (2002).

[9] S. Dev, S. Kumar, S. Verma, and S. Gupta, Phys. Rev. D 76, 013002 (2007).

[10] S. Dev, Sanjeev Kumar, Surender Verma, and Shivani Gupta, Nucl. Phys. B784, 103 (2007).

[11] Luis M. Cebola, David Emmanuel-Costa, and Ricardo Gonzalez Felipe, Phys. Rev. D 92, 025005 (2015).

[12] S. Kaneko, H. Sawanaka, and M. Tanimoto, J. High Energy Phys. 08 (2005) 073.

[13] S. Dev, S. Verma, and S. Gupta, Phys. Lett. B 687, 53 (2010).

[14] E. I. Lashin and N. Chamoun, Phys. Rev. D 78, 073002 (2008).
[15] S. Dev, S. Verma, S. Gupta, and R. R. Gautam, Phys. Rev. D 81, 053010 (2010).

[16] I. Esteban, M. C. Gonzalez-Garcia, M. Maltoni, I. MartinezSoler, and T. Schwetz, J. High Energy Phys. 01 (2017) 087.

[17] F. Couchot, S. Henrot-Versillé, O. Perdereau, S. Plaszczynski, B. Rouillé d'Orfeuil, M. Spinelli, and M. Tristram, Astron. Astrophys. 606, A104 (2017).

[18] C. Jarlskog, Phys. Rev. Lett. 55, 1039 (1985).

[19] J. F. Nieves and P. B. Pal, Phys. Rev. D 36, 315 (1987).

[20] J. F. Nieves and P. B. Pal, Phys. Rev. D 64, 076005 (2001).

[21] J. A. Aguilar-Saavedra and G. C. Branco, Phys. Rev. D 62 , 096009 (2000).

[22] Z.-z. Xing and J.-y. Zhu, Nucl. Phys. B908, 302 (2016).

[23] J. N. Ng and A. N. Kamal, Phys. Rev. D 18, 3412 (1978).

[24] J. Abad, J. G. Esteve, and A. F. Pacheco, Phys. Rev. D 30, 1488 (1984).

[25] L. S. Littenberg and R. E. Shrock, Phys. Rev. Lett. 68, 443 (1992).

[26] M. Doi, T. Kotani, and E. Takasugi, Prog. Theor. Phys. Suppl. 83, 1 (1985).

[27] T. S. Kosmas, G. K. Leontaris, and J. D. Vergados, Prog. Part. Nucl. Phys. 33, 397 (1994).

[28] M. Flanz, W. Rodejohann, and K. Zuber, Eur. Phys. J. C 16, 453 (2000). 
[29] M. Flanz, W. Rodejohann, and K. Zuber, Phys. Lett. B 473, 324 (2000).

[30] W. H. Furry, Phys. Rev. 56, 1184 (1939).

[31] S. Verma, Mod. Phys. Lett. A 31, 1650040 (2016).

[32] S. Dev and S. Kumar, Mod. Phys. Lett. A 22, 1401 (2007).

[33] S.-F. Ge and M. Lindner, Phys. Rev. D 95, 033003 (2017).

[34] W. Rodejohann, J. Phys. G 39, 124008 (2012).

[35] A. Gando et al. (KamLAND-Zen Collaboration), Phys. Rev. Lett. 110, 062502 (2013).

[36] A. Gando et al. (KamLAND-Zen Collaboration), Phys. Rev. Lett. 117, 082503 (2016); 117, 109903(E) (2016).

[37] M. Agostini et al. (GERDA Collaboration), Phys. Rev. Lett. 111, 122503 (2013).

[38] D. R. Artusa et al. (CUORE Collaboration), Eur. Phys. J. C 74, 2956 (2014).
[39] D. R. Artusa et al. (CUORE Collaboration), Adv. High Energy Phys. 2015, 1 (2015).

[40] F. Granena et al. (NEXT Collaboration), arXiv:0907.4054.

[41] J. J. Gomez-Cadenas et al. (NEXT Collaboration), Adv. High Energy Phys. 2014, 1 (2014).

[42] M. Auger et al. (EXO Collaboration), Phys. Rev. Lett. 109, 032505 (2012).

[43] J. B. Albert et al. (EXO-200 Collaboration), Phys. Rev. Lett. 120, 072701 (2018).

[44] A. Abada, V. D. Romeri, M. Lucente, A. M. Teixeira, and T. Toma, J. High Energy Phys. 02 (2018) 169.

[45] Z.-z. Xing, Phys. Rev. D 87, 053019 (2013).

[46] Z.-z. Xing and Y.-L. Zhou, Phys. Rev. D 88, 033002 (2013).

[47] F. Capozzi, E. Lisi, A. Marrone, D. Montanino, and A. Palazzo, Nucl. Phys. B908, 218 (2016). 